www.mdpi.com/journal/socsci

Review

\title{
Prostate Cancer Disparities throughout the Cancer Control Continuum
}

\author{
Meghan E. Borysova ${ }^{1,2}$, Dawood H. Sultan ${ }^{1,2, *}$, Ganna Chornokur ${ }^{2}$, Kyle J. Dalton ${ }^{2}$ and \\ Adewale Troutman ${ }^{3}$ \\ 1 University of South Florida, College of Public Health, Department of Health Policy and \\ Management, 13201 Bruce B. Downs Blvd, MDC 56, Tampa, FL 33612, USA; \\ E-Mail: mborysov@health.usf.edu \\ 2 The Center for Equal Health, University of South Florida, Morsani College of Medicine, 12901 Bruce \\ B. Downs Blvd, MDC 13, Tampa, FL 33612, USA; E-Mails: ganna.chornokur@moffitt.org (G.C.); \\ kdalton@health.usf.edu (K.J.D.) \\ 3 University of South Florida, College of Public Health, Public Health Practice Program, 13201 \\ Bruce B. Downs Blvd, MDC 56, Tampa, FL 33612, USA; E-Mail: atroutm1@health.usf.edu (A.T.) \\ * Author to whom correspondence should be addressed; E-Mail: dsultan@health.usf.edu; \\ Tel.:+813-396-9390.
}

Received: 10 September 2013; in revised form: 29 October 2013 / Accepted: 30 October 2013 / Published: 8 November 2013

\begin{abstract}
Prostate cancer ( $\mathrm{PCa}$ ) is the most commonly diagnosed malignancy and the second leading cause of cancer deaths among men in the United States. The American Cancer Society estimates that 238,590 U.S. men will develop PCa and 29,720 men will die from the disease in 2013. PCa exhibits the most profound racial disparities of all cancers with African American men having a 70\% higher incidence rate and more than two times higher mortality rate than Caucasian men. Published research on PCa disparities focuses on singular outcomes such as incidence, mortality or quality of life. The objective of this paper is to provide a comprehensive summary of the racial disparities found at each stage of the PCa Care Continuum which includes prevention, detection, treatments, and outcomes and survival. It focuses primarily on disparities among Caucasian (white) and African American men.
\end{abstract}

Keywords: prostate cancer; health; disparities; race; African American; cancer control continuum 


\section{Abbreviations}

Prostate cancer, PCa; Digital rectal exam, DRE; prostate specific antigen, PSA; Transurethral resection of the prostate; TURP.

\section{Introduction}

The American Cancer Society estimates that 238,590 men in the United States (US) will develop prostate cancer (PCa) in 2013 and 29,720 men will die from the disease in the same year [1]. These estimates indicate that in 2013 PCa will account for $28 \%$ of all expected new cancer diagnoses and $10 \%$ of all expected cancer deaths [1]. PCa is the most common cancer and the second leading cause of cancer deaths among African American (black) and Caucasian American (white) men. With a 70\% higher incidence rate and more than two times higher mortality rate among black than white men, PCa exhibits the most pronounced racial disparity of all cancers in the US [1].

Since the 1970s the term cancer control continuum has referred to the various stages of cancer research and clinical care. The continuum begins with cancer prevention, followed by detection, treatment, and outcomes and survivorship. The cancer control continuum is a conceptual model through which we can think about the phases of cancer clinical care. This continuum differs from commonly utilized Tumor, lymph Nodes and Metastasis (TNM) cancer staging system which addresses the cellular features and severity of an individual's cancer and may serve as a prognostic indicator.

Racial disparities related to PCa in African Americans have been identified in all four stages of the cancer continuum. While the racial disparities in PCa etiologies are not clearly understood, available evidence shows that disparities exist in a number of factors associated with prevention including diet [2-6] and exercise patterns [7,8], steroid hormone levels [9-12], and vitamin D status [13-16]. Racial disparities are also evident in PCa detection. Racial differences are found in age of diagnosis [1,17], knowledge of risk [18,19], and disease state at clinical presentation [20-25]. Several reports have emphasized racial disparities in the utilization of various treatment strategies including receipt of radical prostatectomy, watchful waiting [26-33], and hormone therapy [32,34]. Outcomes and survival disparities among black and white American men are striking and clearly demonstrated in significant differences in mortality rates [1,17], quality-of-life [35], and end-of-life care [36,37].

The objective of this review is to provide an up to date summary of research findings on the disparities in PCa throughout the four stages of the cancer control continuum with a specific focus on African Americans and Caucasian Americans. The selection of these two racial groups is due to the abundance of research evidence which compared their health and disease outcomes and the vast evidence which shows significant disease prevalence, detection, treatment and survival outcome disparities among them. We intentionally excluded studies which defined black and white American racial groups in terms inclusive of Hispanics or other ethnicities. Nevertheless, some of the studies we reviewed (see [2,3]) relied on black and white American self-identification without explicitly indicating whether or not the racial identity terms used included Hispanic-whites or Hispanic-blacks. We considered black and white American self-identification in these articles to be indicative of non-Hispanic black and non-Hispanic white racial definitions. 
Statistical evidence shows that African American men have not benefited from observed declines in PCa mortality and that black-white disparities exist in PCa incidence and mortality. These persistent realities created a need to provide up-to-date road maps for clinicians and researchers to be able to identify where along the PCa control continuum disparities continue to exist in order to be able to address them. While this is not a systematic review which has a list of defined criteria and statistical analysis for comparative assessments, this article provides a comprehensive up-to-date review which considers the most critical points of PCa knowledge at each point along the PCa control continuum. It differs from previous systematic reviews of PCa topics by identifying gaps in scientific knowledge about a particular component of the PCa control continuum as literature pertaining to that component was being reviewed. That is, summaries of knowledge gaps and discussions of disparities at a specific point along the PCa control continuum are found in the same section of the article. We propose that understanding disparities within each stage of the cancer control continuum is critical for eradicating disparities and reducing the overall prostate cancer burden and disparities among white and black men. We also propose that tying together the disparities reviews and discussions of knowledge gaps at each stage of the PCa control continuum is a more efficient way for organizing ideas.

\section{Methods}

The review is organized in a way which corresponds to the different components of the PCa control continuum. As such, it starts by outlining findings on prevention, followed by the findings on detection, treatment and outcomes and survival. It ends with a number of recommendations for future PCa-related research and clinical care practice.

The articles reviewed were gathered over a period of sixty consecutive days from four databases: PubMed, ScienceDirect, JSTOR and Google Scholar. Prior to the search for articles in the databases, the authors created a set of general conceptual categories to be used for the identification of appropriate search terms. Papers containing one or more of the following search terms were collected: cancer in African Americans, cancer in black Americans, cancer in white Americans, cancer in Caucasian Americans, prostate cancer, prostate cancer control continuum, prostate cancer disparities, prostate cancer in black men, prostate cancer in African American men, prostate cancer in white men, prostate cancer in Caucasian men, prostate cancer in black non-Hispanic men, prostate cancer in white non-Hispanic men, prostate cancer prevention, prostate cancer detection, prostate cancer treatment, prostate cancer outcomes, prostate cancer survival.

At the end of the sixty-day period, the collected papers were initially reviewed for a determination of the ethnic classifications their authors used. Papers were excluded from review if the samples used in their analysis included Hispanic-black, or Hispanic-white, or both as research participants. Papers that used multistage disease progression models as conceptual frameworks and involved diagnoses that were part of molecular and cellular analyses of cancer were also excluded, mainly due to their inappropriateness for a study of the cancer control continuum. The remaining papers were examined to identify which stage(s) of the PCa control continuum and which critical variables at each point along the continuum were included in the analysis. While stage and critical variable identification was conducted, the types of gaps in scientific knowledge at each stage in the PCa control continuum were noted. 
Three of the coauthors reviewed articles on three components of the PCa control continuum. One was tasked with reviewing articles on detection, another was charged with performing reviews on treatment, and a third completed reviews on outcomes and survival. The remaining two coauthors jointly reviewed literature on prevention and carried out global reviews of all of the collected literature and the individual reviews conducted by the three coauthors. This two-stage review process differed slightly from the standard practice in systematic literature reviews, where authors individually reviewed all the articles and subsequently met to determine where they agreed and differed on articles to be included and excluded from the final review. However, our objective was to create a comprehensive and up-to-date review of literature on PCa control continuum and not a systematic review in the fullest technical terms (inclusive of statistical analyses and comparative assessments). Hence, though our method deviated from the established technique for a systematic literature review, it allowed for efficient management of vast literature.

\section{Prevention}

Several factors are thought to contribute to PCa disparities including nutrition, obesity, exercise, steroid hormone levels, and vitamin $\mathrm{D}$. These variables have the potential to be modulated through lifestyle choices and chemoprevention in order to reduce the incidence of PCa particularly among African American men. However, roles for each of these factors in PCa disparities are still being examined and defined.

\subsection{Diet}

Heterocyclic amines, which are carcinogens formed in red meat when cooked at high temperatures have been shown to be consumed at much higher rates by African Americans than Caucasians [4,5]. A relationship between consumption of animal fat, particularly red meat intake, and an increased diagnosis of PCa [38], particularly among African American men is mechanistically feasible and is supported by several lines of research [2,3] but has not been firmly established. A contradictory study indicates that preferences for well-done versus medium or rare-cooked red meat do not correlate with the risk of PCa in any of five racial and ethnic groups examined [39]. However, food preparation preferences including the duration of cooking and cooking temperatures have not been evaluated across racial and ethnic groups. As such it is not clear whether differences in food preparation preferences of black and white males account for PCa incidence disparity.

Consumption of fish has been inversely correlated with PCa risk [38], and is thought to be related to dietary explanations for low PCa rates among Asians [40] whose diet is characterized by high intake of sea/marine foods. However, to our knowledge fish consumption has not been examined for associations with increased rates of PCa among African Americans. Likewise, soy bean and soy bean products are common in the diet of Asians in Asia and in the United States, the groups who exhibit the lowest frequencies of PCa [1,17]. The phytoestrogen activity of isoflavones (organic compounds found in soy bean) is thought to be protective against PCa [6]. Purified soy isoflavones are currently being evaluated in a clinical trial for their potential to reduce PCa in Caucasian and African American men [41]. 
Obesity has long been considered a possible risk factor for PCa. However, studies which examined the associations of obesity, as indicated by body mass index, and the occurrence of PCa have yielded mixed results. Much of the data on obesity and PCa suggests that body mass index itself does not contribute to PCa [42,43] and therefore is not an etiological basis for PCa disparities. However, more recent studies provide data that have demonstrated an association of high-grade PCa, PCa progression, and PCa mortality with obesity [44-46]. Obesity has also been shown to be correlated with lower rates of PCa relapse-free survival and poor tumor characteristics among African American men, but not Caucasian American men [45].

\subsection{Exercise}

Epidemiological studies indicate an inverse relationship between exercise and advanced prostate tumors in men $[47,48]$ and a transgenic mouse model has shown that exercise is associated with a reduced grade of prostatic intraepithelial neoplasia (PIN), the precursor lesions of PCa [49]. In two studies designed to cross-racially evaluate the association of exercise with an increased risk of PCa, physical activity in white men was not shown to provide a protective effect $[7,8]$. However, increased exercise in black men was related to a 35\% lower risk of PCa [8]. Physically inactive African American men are 3.6 times more likely to be diagnosed with PCa than active African American men [7]. This significant disparity may be a result of the presence of lower levels of tumor promoting growth factors among African American men who exercise [50].

\subsection{Steroid Hormones}

Scientific research strongly links steroid hormone levels to the normal maturation of the prostate. Studies have shown that androgen (a male sex hormone) deprivation therapy reduces tumor burden. This indicates that increases in androgens may play a role in slowing tumor progression and may be involved in tumor development. However, the roles of androgens, particularly testosterone and dihydrotestosterone, in tumorigenesis and PCa prevalence disparities have not been clearly demonstrated [9]. It should also be noted that there are recent reports which contradict the findings of previous research and suggest that low androgen levels may promote tumor growth more than high levels [51-53]. However, in contrast, several researchers have also argued that African American men, relative to Caucasian and Asian American men, have significantly higher levels of testosterone or dihodroxytestosterone, thus contributing to the higher rates of PCa incidence among US black men. Yet, despite the mechanistic feasibility that androgen levels mediate the occurrence of PCa, strong epidemiological evidence on the exact role of androgens in PCa disparities is lacking. Estimates derived from the cumulative data currently available are inconclusive and as such, the findings on the roles of androgen levels in PCa etiology are mixed. Furthermore, available measures of serum testosterone and dihodroxytestosterone levels do not indicate that African American men have higher levels of androgens [54]. However, androgen levels are most frequently measured in adult males around the time of PCa diagnosis. Yet, several studies have shown that androgen levels are higher in African American men than in Caucasian men during infancy, early life and young adulthood [10-12].

The androgen receptor mediates the effects of androgen. The common genetic polymorphisms CAG and GGN repeats in the androgen receptor gene are by length inversely related to the levels of 
testosterone produced [55]. While some evidence suggests that the numbers of repeats vary by race with African American men having fewer repeats than Caucasians [9] (and thereby higher levels of testosterone) data from a study exclusively evaluating African American men shows no evidence of a relationship between the number of repeats and their PCa risk [56]. Also, preliminary data from the isoflavones-PCa prevention trial [41] (reported earlier) indicate that reduction in normal androgen levels may protect against low-grade PCa and showed no differences between white and black men. However, the enrollment rate of African Americans in this trial was $4 \%$ at the time of this review. Current trials are ongoing to further explore this possibility. Regardless, uncertainty about research findings suggests that future studies are needed in order to determine if significantly higher levels of early life androgens in black men significantly contribute to the observed white-black PCa incidence and prevalence disparities in the US.

\subsection{Vitamin $D$}

Vitamin D is involved in cellular differentiation, proliferation and metastases [57]. Vitamin D deficiency has been epidemiologically linked to several cancers [58,59]. Vitamin D levels are significantly higher in Caucasian Americans than in African Americans [13,14], largely due to the lower absorption of UV rays in the densely pigmented skin of black people [60]. In one study of African American men, 61\% were deficient in vitamin D (hypovitaminosis D) [61]. The link between vitamin D deficiency and the higher incidence of PCa among African American men is mechanistically plausible and may have some biological basis $[15,16]$. However, no correlation was found between vitamin D status in middle-aged or elderly professional black men and PCa risk [62]. Future studies are required in order to determine if vitamin D status at birth or throughout early life poses a lifelong higher risk of PCa among black men.

\section{Detection}

Detection is the stage of the cancer control continuum in which PCa risk is assessed, screening is performed, and PCa detection is confirmed. Not only are African American men 1.4 times more likely to be diagnosed with PCa [1], but racial and ethnic disparities exist in each sub-stage of detection, including risk assessment, screening and ultimately the determination of the presence of cancerous cells in the prostate [1].

\subsection{Risk Assessment}

Race, age, and family history are the three well-established risk factors for PCa. Self-reported African American heritage is associated with a higher risk for PCa. Every decade of age increases the lifetime risk of PCa diagnosis for men of all races. However, disparities in age-related risk of PCa incidence between black and white American men of the same age range are significant. The highest age-related disparity in incidence is in the fourth decade of life (between the ages of 30 and 39). In this age bracket African American men have a 2.8 times higher likelihood of developing PCa than their white counterparts [1,17]. In every subsequent decade of life African American men have significantly higher prevalence rates of PCa than white men. However, the disparity gradually decreases with 
increases in age. The reasons for this trend have not been defined and future research efforts identifying the causes of the age-incidence disparity and its decline will help in understanding the overall etiologies of racial disparities in PCa.

Also, family history has been well documented as a risk factor for PCa [54,63]. Research evidence shows a 2-4 times more risk if one first degree male relative has had PCa and a five to eleven times more risk if two or three first degree male relatives have had PCa [1]. Family history is widely utilized as part of risk assessment for PCa and is credited with prompting many men to seek PCa screening. However, knowledge among African Americans regarding family history as a risk factor for PCa has been demonstrably lower than in white Americans [18,19]. Therefore, lack of knowledge is considered a factor that impedes black men's decision to be screened prior to evident symptoms.

\subsection{Screening}

Screening is the attempt to look for PCa in an early, subclinical stage, and before it is causing any symptoms. Currently, the Prostate Specific Antigen (PSA) serum test and a Digital Rectal Examination (DRE) are the only two clinically approved methods for PCa screening. Although screening has dramatically reduced PCa mortality, numerous racial disparities in PCa screening have been reported. Studies have shown that African American men are less likely to undergo screening due to lack of health insurance [64], a mistrust of traditional healthcare [65], and reduced access to primary health care [66]. Apart from an increased effort to provide prostate cancer screening, there is currently no consistent strategy to address disparities in prostate cancer treatment outcomes. Screening and early detection are fronted in the literature as factors that would contribute to reductions in prostate cancer mortality. However, there is little research evidence which supports a definitive conclusion that screening actually reduces PCa-related deaths [67]. While the uncertainty exists about the effectiveness of the screening techniques for prostate cancer, research conclusions often encouraged patients to obtain from their healthcare providers information about the risks, benefits and potential harms of screening. Such information is considered instrumental in facilitating an informed decision making (IDM) which incorporates personal values and preferences [68]. However, along with mistrust and perceptions of discrimination and racism, poor patient-provider interaction has been found to be a factor which impedes PCa IDM among racial and ethnic minority groups. This is particularly so in four aspects of IDM in PCa screening: individual risk assessment, consideration of individual values and beliefs, knowledge of the risks and benefits of screening modalities, and consideration of age and lifespan health issues [69-72].

\subsection{Biopsies}

Biopsies are the only tests which can fully confirm the diagnosis of PCa. They are offered to men for whom PSA testing and DRE results indicate prostate status abnormalities. African American men are generally three years younger at the time of PCa diagnosis [20]. They present at clinic with higher PSA values [24], larger tumor volumes [23], more aggressive PCa disease [21,22] and higher rates of biochemical recurrence than their white counterparts [25]. The reasons for these racial disparities are unknown. Some studies reported that older African American men are somewhat more likely to have their PCa unstaged by pathology than Caucasian American men [73]. However, this disparity was 
significantly reduced with the introduction of new staging guidelines in 1995 [74]. Yet, observed differences in PSA values [24], tumor volumes [23], PCa disease severity [21,22] and rates of biochemical recurrence [25] are still not adequately explained.

\section{Treatment}

Choosing the right treatment is one of the best known ways to prolong survival and improve the quality of life after PCa diagnosis. To battle PCa effectively, clinicians and patients must consider several factors related to efficacy, side effects, treatment cost, and personal preferences. In the process, patients and clinicians often consider a number of alternatives related to disease management and treatment modalities.

\subsection{Watchful Waiting (Also Known as Expectant Management and Active Surveillance)}

Due to the indolent nature of subclinical prostate cancers and the significantly higher odds of serious side effects of most therapies, it may be beneficial for a patient to defer treatment and choose active surveillance to closely monitor a low-grade, prostate-confined cancer until clinical symptoms of progression appear [75]. Watchful waiting is also used in older men or men with co-morbid conditions for whom aggressive therapies may not be well-tolerated or may not significantly promote longevity [31]. Recent studies indicate that African American men are about 1.4 times more likely to undergo expectant management than white men with the same diagnosis. However, after adjustments were made for age, co-morbidity, stage at diagnosis, PCa grade and life expectancy, available research evidence suggests that African-American men are monitored less closely and have fewer doctor visits compared to white patients [26,76]. Current research evidence also indicates that African American men are six (6) times more likely not to have any medical monitoring visits or clinical procedures during the 60-month post-diagnosis follow-up period compared to white American men [26]. We note that the overall impact of these disparities on survival is currently not fully understood.

Furthermore, the American Urological Association (AUA) provided guidelines on the detection of PCa which were based on a systematic literature review of more than 300 studies which examined PCa, PCa incidence and mortality, PCa health-related quality of life, PCa diagnostic accuracy and harms of PCa testing. The AUA's conclusions which upgraded screening age to the 55-69 bracket ran against standard practice recommendations for screening black men at a much younger age [77]. However, the AUA recommendations were consistent with the findings of an earlier study by the U.S. Preventive Services Task Force (USPSTF) which advised against PSA-based screening for PCa [78]. The USPSTF concluded that benefits of PSA-based testing for PCa do not outweigh clinical harms which could potentially result from the test itself [78]. The PSA has potential for false-negative and false-positive results. However, these recommendations might be technically problematic in the case of African American (black) men. They do not account for specific clinical characteristics of African American (black) PCa patients. Previous studies clearly demonstrated that African American men are generally three years younger at the time of PCa diagnosis [20] and that they present at clinic with higher PSA values [24], larger tumor volumes [23], more aggressive PCa disease [21,22] and higher rates of biochemical recurrence than their white counterparts [25]. Though the AUA and USPSTF studies were based on extensive literature reviews, results of the studies we reviewed force us to 
believe that the new guidelines and recommendations have to be tempered and reconsidered in the specific case of African American (black) men who have a clinical record and profile which differentiates them from white men or men of other ethnic identifications [21-23].

Also evidence which shows that black men have not benefited from the current declines in prostate cancer mortality [1] is another reason which forces us to suggest a careful consideration of the current guidelines as well as the choice of watchful waiting as common clinical practice when treating black PCa patients [26]. The findings of earlier studies suggest that among men who have advanced or aggressive PCa, active therapy is the best treatment choice [79]. Recent studies suggest that younger age and greater PCa severity at diagnosis are significant predictors of progression to treatment [80,81]. As we have noted earlier in this article, these are some of the clinical characteristics which distinguish black PCa patients. A careful investigation of whether or not active surveillance is a contributor to increased PCa mortality risks among black men and black-white PCa mortality disparities is, therefore, warranted.

\subsection{Radical Prostatectomy}

Radical prostatectomy (RP), which involves the removal of the prostate gland and some of the tissue around it, is typically administered in patients with localized tumors and no evidence of metastases. Although use of radical prostatectomy as a curative alternative is increasing in all racial groups, African American men have significantly lower odds of being treated with this surgery than white men [82]. Studies show that after controlling for covariates [51,83], prescription of radical prostatectomy highly correlates with higher socioeconomic status [28,84], type of health insurance [29], treatment in a county or private hospital, educational level [29] and marital status [84]. Unmarried African American men with lower socio-economic and educational status and who have no insurance or Medicare are less likely to receive radical prostatectomy. This is regardless of age, co-morbid conditions and cancer stage [29,84]. This disparity is thought to result from either the patient's personal bias against radical prostatectomy [31] or implicit racial discrimination against African American patients by physicians [31,32].

\subsection{Radiation Therapy}

Radiation therapy includes brachytherapy or external radiation treatment (XRT). Brachytherapy involves the insertion of radioactive seeds into the tumor tissue and is recommended for treatment of early T1 or T2-staged prostate-confined cancers. XRT is recommended for later staged tumors that have spread, and involves directed high-energy X-ray radiation of the tumor from an external source. Studies suggest that African American men (31\%) are more likely than white men (19\%) to choose radiation over radical prostatectomy [33]. Whether this treatment choice affects disparities in survival is currently unknown.

\subsection{Hormone Therapy}

Hormone therapies such as luteinizing hormone-releasing hormone (LHRH) agonists are a form of chemical castration to reduce androgen levels and are used as palliative treatments for advanced disease. Use of hormone therapy is shown to improve cancer survival [85]. LHRH utilization among 
African American patients with late-stage metastatic PCa is reportedly lower than among white patients. Recent estimates indicate that $77.6 \%$ of white PCa patients receive hormonal therapy before death. In contrast, only $71 \%$ of African American PCa patients are reported to have similar treatment before death [85]. Of the white American men who received hormonal therapy, 71\% received it early (within 4 months of diagnosis) while only 58.3\% of African American men received early treatment. Furthermore, African American men were more than twice as likely as white men to receive delayed hormonal therapy (12.7\% and 6.2\%, respectively) [85].

\subsection{Chemotherapy}

Chemotherapy is used for salvage treatment in hormone refractory PCa or for advanced PCa with distant metastasis. It has been shown to extend the duration and quality of life in some patients [86]. Chemotherapy for PCa management is a relatively new approach to increasing survival and racial and ethnic disparities in the use of chemotherapy for PCa management have not been reported.

\section{Outcomes/Survival}

The PCa mortality rate, an outcome of disease progression and disease-related death, is more than two times higher among African American men than white men [1,17]. In this section we will address racial and ethnic disparities in PCa outcomes and survivorship and their links to PCa-specific mortality, PCa patient quality-of-life, and end-of-life care for PCa patients.

\subsection{Mortality}

PCa tumor grade and stage have repeatedly been reported to be higher at clinical presentation among African American men than white men [21,22]. Tumor stage is a prognostic factor for survival [87]. The extent to which tumor stage contributes to the significantly higher PCa mortality rate among African American men is not clearly understood. Tumor stage at diagnosis, initial treatment, and socio-economic status reportedly account for the majority of the difference in survival rates among African American and white PCa patients [88,89]. However, unidentified factors are thought to contribute to an increased mortality rate and a significant decrease in life expectancy among African Americans with advanced stage PCa compared to patients from all other racial and ethnic groups. A recent study indicated that PCa mortality rates within the 1-year survival or 3-year survival periods were not different among white and black Medicare recipients when treated exclusively in National Cancer Institute (NCI) cancer centers [90]. This suggests that specialized/higher quality of PCa care may remove short-term survival endpoint disparities. Yet, the relative contribution of health care following treatment on survival disparities is still unclear and must be further examined in order to generate measures which could aid in efforts to reduce PCa mortality among African American men.

Though many of the factors contributing to the higher PCa disease and mortality burdens among black American men are still unidentified, obesity has been shown to positively correlate with a higher stage of PCa [45,91,92] in men of all races and is a significant predictor of overall and PCa-specific mortality [93,94]. Data suggest that obesity, independent of Gleason score upon biopsy [95], is a risk factor for biochemical relapse in African American men following radical prostatectomy [45,96]. 
Obesity is often linked to food consumption habits and preferences. Yet, a recent study shows no effect of processed versus unprocessed red meats on PCa progression after diagnosis. However, the study revealed more than 2-4 times increased risk of PCa recurrence or progression with increased consumption of eggs and poultry meat [97].

\subsection{Quality of Life}

Issues related to the quality of life of PCa survivors are increasingly gaining attention. Radical prostatectomy and radiation, the two curative PCa treatments, have numerous adverse effects which often diminish the quality of life of PCa survivors. In comparison with white men, African American men who undergo curative treatment consistently report a lower quality of life related to physical and psychological symptoms [35]. Reported PCa treatment effects included decreased emotional wellbeing and depression [98], subjective stress [99], higher levels of treatment decisional regret [83], increased number of erectile, urinary and bowel dysfunctions [33,100], sleep disturbances [101], and high levels of pain [102]. Studies indicate that following PCa treatment African American men who maintain close emotional relationships with their intimate partners report a higher quality of life despite the major side-effects of treatment (urinary, bowel and sexual dysfunctions) [100]. This reinforces the importance of emotional wellbeing and its influence on the physical side effects of PCa treatment. In addition, it has been reported that spirituality and having a personal relationship with God plays an important role in the lives of African American PCa cancer survivors [103].

\subsection{End of Life}

End-of-life care focuses on psychosocial support and the treatment of pain and other symptoms associated with terminal illness [104]. It is considered a deeply meaningful and important stage for PCa patients and their families. Though end-of-life care is sometimes provided by family members or partners, in the US it is frequently provided by hospice. The appropriate use of hospice care has been shown to improve the quality of life of terminally ill cancer patients [105]. However, hospice care is utilized less frequently by African Americans [36]. African American patients in end-of-life care more frequently die in hospitals than do white American patients [37]. Recent evidence suggests that the lower rates of hospice use by African Americans and minorities may be due to inadequate medical infrastructures and support services in minority neighborhoods [106].

\section{Future Research and Practice Recommendations}

Eliminating racial disparities in PCa requires research that will identify the causes of the disparities within each stage of the cancer control continuum. Current and past research has largely focused on studies of socioeconomic and environmental factors and biological contributions to racial disparities. Studies from the rapidly emerging field of life-course health outcomes which predicts a causal relationship between early life exposures and the development of prostate tumors are expected to add new insights into the highest burden of PCa morbidity and mortality among African American men. A considerable volume of previous research findings have guided the design of a variety of PCa prevention and treatment interventions. However, available research evidence indicates that interventions 
and clinical trials which focus on a singular disparity, such as screening, will not alone eliminate the overall disparities in incidence or mortality. Yet, clinical trials that address PCa prevention, detection, treatment, outcomes and survival across different racial and ethnic groups are needed. A few are currently being conducted. An initiative to collect clinical data on men from birth throughout life would ultimately reveal many answers to life-course questions pertaining to PCa disparities. In-depth individual genetic, epigenetic, and lifestyle profiles should be developed to replace race as a factor of risk. And, a sensitive, specific, accessible, reliable screening tool, tested and proven to have high efficacy in African American men, is needed in order to improve the utility of screening and screening rates. Several ongoing trials which include assessments of stratification by race are focused on PCa screening. Currently there are several clinical trials assessing exercise or obesity and PCa progression or outcomes. For instance, an ongoing PCa clinical trial is examining the relationship between heterocyclic amine exposure in African American men and PCa screening results. Vitamin D intervention trials will help reveal causation and potential effects of vitamin $\mathrm{D}$ on reducing racial and ethnic PCa disparities. The use of soy isoflavones has been demonstrated to reduce PCa biomarkers in a 12-week intervention among PCa patients, and a current clinical trial is testing the efficacy of soy isoflavones specifically among African American men. However the trials do not specifically stratify by race and do not require a minimum number of African American men to be recruited. As such their outcomes will be subject to debate and are unlikely to have the desired overall effect to reduce PCa racial disparities.

The reasons for the disproportionately lower rates of radical prostatectomy and higher rates of a watchful waiting among African Americans ought to be established and reliable evidence of survival benefits in patients receiving radical prostatectomy versus watchful waiting should be cross-racially determined. However, current research evidence suggests that an intervention to provide more treatment options and information to African American men and their families may help reduce racial disparities in PCa mortality. Therapies which may improve outcomes in African American men with low grade PCa and who have elected active clinical surveillance need to be researched. This is of particular significance due to the high watchful waiting rates among African American PCa patients. Additional basic science and clinical research testing the efficacy of chemotherapy and response times of an androgen ablation in African American men with high grade PCa is also needed, and histological or genetic tumor markers need to be developed to predict the aggressive potential of early prostate-confined tumors.

Two active clinical trials which were examined in this study explore quality-of-life issues in African American men diagnosed with PCa. One trial aims to establish and validate the Memorial Anxiety Scale for PCa in African American men diagnosed with PCa and to explore the prevalence of distress, anxiety, and depression in this population. The other trial aims to develop effective ways to help both African American PCa survivors and their intimate partners to cope with the problems and challenges experienced after radical prostatectomy. While both clinical trials address important issues of mental and psychological aspects of coping with PCa, we note that no clinical trial is designed to reduce the side effects of treatment in order to improve quality of life. Nor are there any trials attempting to determine if African American men indeed have a higher likelihood of clinical side effects following surgery or radiation. These gaps in practice and knowledge require closing as a measure to reduce racial disparities in PCa quality of life. 
Finally, intervention strategies to improve end-of-life care and hospice use for minority populations are necessary, and research into the attitudes of physicians in the prescription of hospice as well as patient attitudes across different races and ethnicities are needed. Beyond clinical trial-based interventions, other interventions which promote informed decision making in PCa screening are also needed. However, current evidence indicates that to be successful, these interventions should accommodate the psychological predispositions of African American men, their mistrust of medicine and clinicians, and their general reticence about scientific research.

\section{Conclusions}

This article provides an up-to-date comprehensive review of literature on black and white racial disparities along the PCa control continuum. The review outlined disparities in prevention, detection, treatment, outcomes and survival, and how these disparities are linked to PCa mortality. It reported research findings which revealed that black men were younger at the time of PCa diagnosis, and that they were more likely to present at clinic with higher PSA values, larger tumor volumes, more aggressive PCa disease, and higher rates of biochemical relapse than white men. In light of these findings, and the fact that black men currently experience a 70\% higher PCa incidence rate and more than two times higher PCa mortality rate than white men, the review questioned the appropriateness of recent guidelines and recommendations on active surveillance. Also, examination of disparities at specific points along the PCa control continuum allowed for easy identification of gaps in scientific knowledge at each point. Tying together the disparities, reviews and discussions of knowledge gaps at each stage of the PCa control continuum, provided a more efficient way for organizing ideas. These are features which distinguish this review from other reviews of PCa disparities.

However, this review is not without limitations. It lacks the analytical rigor which is achieved in systematic literature reviews by statistical analysis and statistically driven comparative assessments. Also, some of the articles we reviewed did not provide clear distinction of whether or not black and white ethnicity definitions were exclusive of Hispanic-black and Hispanic-white. In addition, this review did not include literature on Hispanic (Latino) American men or men of other ethnicities (e.g., Asian, Middle Eastern). And, more importantly, the scope of this article did not allow for a comprehensive assessment of how socioeconomic status variables are linked to each of the different stages in the PCa control continuum.Furthermore, current discourses on African American ethnicity often point to the heterogeneity of the black American male population which includes not only black men born in the US, but also immigrant black men from the Caribbean and continental Africa. Though this article did not review research findings on PCa that are relevant to Afro Caribbean and continental African men in the US, a growing literature suggests that some of the PCa experiences of Afro Caribbean men show remarkable similarities with the general experiences of African American men. Afro Caribbean men in the US and African American men tend to have similar PCa screening behaviors that are significantly different from the behaviors of their white male counterparts. For instance, studies which included African American and Afro Caribbean men living in the US showed that they had greater PCa worry than white men. They were also less likely to maintain annual PSA screening and were more afraid of PSA tests, PSA screening scores, and DRE examination than white men. Even after adjustments were made for socioeconomic status, access to healthcare, and 
comorbidities, African American and Afro Caribbean men living in the US were still less likely to undergo DRE screening than white American men. Also, among African American and Afro Caribbean men in the US, screening fear played similar roles in predicting the likelihood of undergoing an initial DRE screening [107-110]. These commonalities imply that these men are likely to have similar PCa prevention and detection profiles. Though studies report differences among Afro Caribbean (in the Caribbean) and African American men in PCa incidence, with Caribbean men of African descent showing significantly higher incidence than African American men [111], there are no studies which examined whether or not migration to the US affected the PCa risk attributable to a gene-environment interaction among black Caribbean men.

Finally, some studies questioned epidemiological surveillance data showing PCa incidence rates in Sub-Saharan African black men that are lower than the rates among white men living in Africa and African American men. Instead these studies indicated elevated PCa incidence among black men in the sub-continent. The studies also revealed that Sub-Saharan African black men present at clinic with larger tumor volumes and more aggressive disease and that regional variations in incidence rates exist, with East Africa having a heavier PCa disease burden [112,113]. Results of a study using data from the International Agency for Research on Cancer (IARC) and the National Cancer Institute Surveillance, Epidemiology, and End Results Program for 1973-2007 suggest that PCa rates among African men have increased between 1987 and 2002. The same study also indicates that though non-distant stage PCa rates in Sub-Saharan black men are lower than those among African American men, the rates of distant stage PCa in East African men are comparable to those among African Americans [113]. We could not find studies comparing Sub-Saharan African men living in the US with either African American men or Afro Caribbean men living in the US. Also, as in the case of Afro Caribbean men in the US, there are no studies which examined whether or not migration to the US affected the PCa risk attributable to a gene-environment interaction among Sub-Saharan African black men. However, a population-based study in the United Kingdom (UK) indicated that male black Caribbean and African immigrants have significantly higher risks of developing PCa than white UK men, and that black Caribbean and African men in the UK have similar PCa rates, suggesting a common genetic etiology [114]. The similarities in PCa risks and rates found in the UK study suggest that the PCa incidence rates among African men living in the US are likely to be higher than rates among African American men. However, at present there is not sufficient literature to enable us to make assumptions about whether or not PCa risk and rate patterns among black African or Afro Caribbean men living in the US will converge to those of African American men, or at what point in time such convergence (if any) might occur. Also, the small number of studies on Afro Caribbean and continental African black

men living in the US did now allow us to provide a comprehensive review of findings on all of the key variables along the entire PCa control continuum that are relevant to these men.

\section{Conflicts of Interest}

The authors declare no conflict of interest.

\section{References}

1. American Cancer Society. Cancer Facts \& Figures 2013. Atlanta: American Cancer Society, 2013. 
2. Carmen Rodriguez, Marjorie L. McCullough, Alison M. Mondul, Eric J. Jacobs, Ann Chao, Alpa V. Patel, Michael J. Thun, and Eugenia E. Calle. "Meat consumption among Black and White men and risk of prostate cancer in the Cancer Prevention Study II Nutrition Cohort.” Cancer Epidemiol Biomarkers Prev 15 (2006): 211-16.

3. Richard B. Hayes, Regina G. Ziegler, Gloria Gridley, Christine Swanson, Raymond S. Greenberg, G. Marie Swanson, Janet B. Schoenberg, Debra T. Silverman, Linda M. Brown, Linda M. Pottern, Jonathan Liff, Ann G. Schwartz, Joseph F. Fraumeni, Jr., and Robert N. Hoover. "Dietary factors and risks for prostate cancer among blacks and whites in the United States.” Cancer Epidemiol Biomarkers Prev 8 (1999): 25-34.

4. Kenneth T. Bogen, and Garrett A. Keating. "U.S. dietary exposures to heterocyclic amines." Journal of Exposure Analysis and Environmental Epidemiology 11 (2001): 155-68.

5. Kenneth T. Bogen. "Cancer potencies of heterocyclic amines found in cooked foods." Food and Chemical Toxicology 32 (1994): 505-15.

6. Norie Kurahashi, Motoki Iwasaki, Manami Inoue, Shizuka Sasazuki, and Shoichiro Tsugane. "Plasma isoflavones and subsequent risk of prostate cancer in a nested case-control study: The Japan Public Health Center.” Journal of Clinical Oncology 26 (2008): 5923-29.

7. Geraldine Clarke, and Alice S. Whittemore. "Prostate cancer risk in relation to anthropometry and physical activity: The National Health and Nutrition Examination Survey I Epidemiological Follow-Up Study.” Cancer Epidemiol Biomarkers Prev 9 (2000): 875-81.

8. Steven C. Moore, Tricia M. Peters, Jiyoung Ahn, Yikyung Park, Arthur Schatzkin, Demetrius Albanes, Albert Hollenbeck, and Michael F. Leitzmann. “Age-specific physical activity and prostate cancer risk among white men and black men.” Cancer 115 (2009): 5060-70.

9. Elizabeth A. Platz, and Edward Giovannucci. "The epidemiology of sex steroid hormones and their signaling and metabolic pathways in the etiology of prostate cancer.” Journal of Steroid Biochemistry and Molecular Bioliology 92 (2004): 237-53.

10. Ronald Ross, Leslie Bernstein, Howard Judd, Rosemarie Hanisch, Malcolm Pike, and Brian Henderson. "Serum testosterone levels in healthy young black and white men.” Journal of the National Cancer Institute 76 (1986): 45-48.

11. R.K. Ross, L. Bernstein, M.C. Pike, B.E. Henderson, R.A. Lobo, F.Z. Stanczyk, and H. Shimizu. "5-alpha-reductase activity and risk of prostate cancer among Japanese and US white and black males.” The Lancet 339 (1992): 887-89.

12. Louis Calistro Alvarado. "Population differences in the testosterone levels of young men are associated with prostate cancer disparities in older men.” American Journal of Human Bioliology 22 (2010): 449-55.

13. Marian T. Hannan, Heather J. Litman, Andre B. Araujo, Christine E. McLennan, Robert R. McLean, John B. McKinlay, Tai C. Chen, and Michael F. Holick. "Serum 25-hydroxyvitamin D and bone mineral density in a racially and ethnically diverse group of men." Journal of Clinical Endocrinology \& Metabolism 93 (2008): 40-46.

14. N. H. Bell, A. Greene, S. Epstein, M. J. Oexmann, S. Shaw, and J. Shary. "Evidence for alteration of the vitamin D-endocrine system in blacks.” Journal of Clinical Investigation 76 (1985): 470-73. 
15. Elizabeth A. Platz, Eric B. Rimm, Walter C. Willett, Philip W. Kantoff, and Edward Giovannucci. "Racial variation in prostate cancer incidence and in hormonal system markers among male health professionals.” Journal of the National Cancer Institute 92 (2000): 2009-17.

16. Edward Giovannucci. "The epidemiology of vitamin D and cancer incidence and mortality: A review (United States).” Cancer Causes Control 16 (2005): 83-95.

17. American Cancer Society. Cancer Facts \& Figures 2009. Atlanta: American Cancer Society, 2009.

18. G. E. Smith, M. J. DeHaven, J. P. Grundig, and G. R. Wilson. "African-American males and prostate cancer: Assessing knowledge levels in the community.” Journal of the National Medical Association 89 (1997): 387-91.

19. Joan R. Bloom, Susan L. Stewart, Ingrid Oakley-Girvans, Priscilla Jane Banks, and Subo Chang. "Family history, perceived risk, and prostate cancer screening among African American men." Cancer Epidemiology, Biomarkers \& Prevention 15 (2006): 2167-73.

20. Mudashiru A. Salami, Blessing Etukakpan, and E. Oluwabunmi Olapade-Olaopa. "Update on prostate cancer in black men.” The Journal of Men's Health \& Gender 4 (2007): 456-63.

21. Richard M. Hoffman, Frank D. Gilliland, J. William Eley, Linda C. Harlan, Robert A. Stephenson, Janet L. Stanford, Peter C. Albertson, Ann S. Hamilton, W. Curtis Hunt, and Arnold L. Potosky. "Racial and ethnic differences in advanced-stage prostate cancer: The prostate cancer outcomes study.” Journal of the National Cancer Institute 93 (2001): 388-95.

22. Ingrid Oakley-Girvan, Laurence N. Kolonel, Richard P. Gallagher, Anna H. Wu, Anna Felberg, and Alice S. Whittemore. "Stage at diagnosis and survival in a multiethnic cohort of prostate cancer patients.” American Journal of Public Health 93 (2003): 1753-59.

23. Ricardo F. Sanchez-Ortiz, Patricia Troncoso, Richard J. Babaian, Josep Lloreta, Dennis A. Johnston, and Curtis A. Pettaway. "African-American men with nonpalpable prostate cancer exhibit greater tumor volume than matched white men.” Cancer 107 (2006): 75-82.

24. Ted O. Morgan, Steven J. Jacobsen, William F. McCarthy, Debra J. Jacobson, David G. McLeod, and Judd W. Moul. “Age-specific reference ranges for prostate-specific antigen in black men.” New England Journal of Medicine 335 (1996): 304-10.

25. Isaac J. Powell, Cathryn H. Bock, Julie J. Ruterbusch, and Wael Sakr. "Evidence supports a faster growth rate and/or earlier transformation to clinically significant prostate cancer in black than in white American men, and influences racial progression and mortality disparity.” The Journal of Urology 183 (2010): 1792-97.

26. Vickie L. Shavers, Martin Brown, Carrie N. Klabunde, Arnold L. Potosky William Davis, Judd Moul, and Angela Fahey. "Race/ethnicity and the intensity of medical monitoring under 'watchful waiting' for prostate cancer.” Medical Care 42 (2004): 239-50.

27. Steven B. Zeliadt, Scott D. Ramsey, David F. Penson, Ingrid J. Hall. Donatus U. Ekwueme, Leonard Stroud, and Judith W. Lee. "Why do men choose one treatment over another?: A review of patient decision making for localized prostate cancer.” Cancer 106 (2006): 1865-74.

28. Matthew R. Cooperberg, Deborah P. Lubeck, Maxwell V. Meng, Shilpa S. Mehta, and Peter R. Carroll. "The changing face of low-risk prostate cancer: Trends in clinical presentation and primary management.” Journal of Clinical Oncology 22 (2004): 2141-49.

29. Beth A. Jones, Wen-Liang Liu, Andre B. Araujo, Stanislav V. Kasl, Stephanie N. Silvera, Hosanna Soler-Vilá, Mary G.M. Curnen, and Robert Dubrow. "Explaining the race difference in 
prostate cancer stage at diagnosis.” Cancer Epidemiology, Biomarkers \& Prevevention 17 (2008): 2825-34.

30. J. Kellogg Parsons, Lorna Kwan, Sarah E. Connor, David C. Miller, and Mark S. Litwin. "Prostate cancer treatment for economically disadvantaged men: A comparison of county hospitals and private providers.” Cancer 116 (2009): 1378-84.

31. Linda C. Harlan, Arnold Potosky, Frank D. Gilliland, Richard Hoffman, Peter C. Albertsen, Ann S. Hamilton, J. W. Eley, Janet L. Stanford, and Robert A. Stephenson. "Factors associated with initial therapy for clinically localized prostate cancer: Prostate cancer outcomes study." Journal of the National Cancer Institute 93 (2001): 1864-71.

32. Kathryn E. Richert-Boe, Sheila Weinmann, Jean A. Shapiro, Benjamin A. Rybicki, Shelley M. Enger, Stephen K. Van Den Eeden, and Noel S. Weiss. "Racial differences in treatment of early-stage prostate cancer.” Urology 71 (2008): 1172-76.

33. Kimberly Peay, Sally Elsamanoudi, Robin Lockhart, Susan Hogue, Thomas E. Novak, and Stephen A. Brassell. "Race, treatment choice, and health-related quality of life in patients undergoing counseling for prostate cancer.” The Journal of Urology 181 (2009): 26.

34. William T. Lowrance, James A. Eastham, David S. Yee, Lindsay M. Jacks, Peter T. Scardino, and Elena B. Elkin. "Racial disparities in the treatment and survival of locally advanced prostate cancer patients.” The Journal of Urology 181 (2009): 27-38.

35. Rosell Jenkins, Leslie R. Schover, Rachel T. Fouladi, Carla Warneke, Leah Neese, Erica A. Klein, Craig Zippe, and Patrick Kupelian. "Sexuality and health-related quality of life after prostate cancer in African-American and white men treated for localized disease.” Journal of Sex and Marital Therapy 30 (2004): 79-93.

36. Beth A. Virnig, A. Marshall McBean, Sara Kind, and Rishi Dholakia. "Hospice use before death: Variability across cancer diagnoses.” Medical Care 40 (2002): 73-78.

37. Alexander K. Smith, Craig C. Earle, and Ellen P. McCarthy. "Racial and ethnic differences in end-of-life care in fee-for-service Medicare beneficiaries with advanced cancer.” Journal of the American Geriatrics Society 57 (2009): 153-58.

38. Moamen Amin, Suganthiny Jeyaganth, Nader Fahmy, Louis R. Bégin, Samuel Aronson, Stephen Jacobson, Simon Tanguay, Wassim Kassouf, and Armen Aprikian. "Dietary habits and prostate cancer detection: A case-control study.” Canadian Urological Association Journal 2 (2008): 510-15.

39. Sangita Sharma, Xia Cao, Lynne R. Wilkens, Jennifer Yamamoto, Annette Lum-Jones, Brian E. Henderson, Laurence N. Kolonel, and Loïc Le Marchand. "Well-done meat consumption, NAT1 and NAT2 acetylator genotypes and prostate cancer Risk: The multiethnic cohort study.” Cancer Epidemiology, Biomarkers \& Prevention 19 (2010): 1866.

40. Mitsuru Mori, Naoya Masumori, Fumimasa Fukuta, Yoshie Nagata, Tomoko Sonoda, Fumio Sakauchi, Hirofumi Ohnishi, Masanori Nojima, and Taiji Tsukamoto. "Traditional Japanese diet and prostate cancer.” Molecular Nutrition and Food Research 53 (2009): 191-200.

41. Nagi B. Kumar, Loveleen Kang, Julio Pow-Sang, Ping Xu, Kathy Allen, Diane Riccardi, Karen Besterman-Dahan, and Jeffrey P. Krischer. "Results of a randomized phase I dose-finding trial of several doses of isoflavones in men with localized prostate cancer: Administration prior to radical prostatectomy.” Journal of the Society Integrative Oncology 8 (2009): 3-13. 
42. Abraham M. Nomura. "Body size and prostate cancer." Epidemiologic Reviews 23 (2001): 126-31.

43. Susan M. Gapstur1, Peter H. Gann, Peter Kopp, Laura Colangelo, Christopher Longcope, and Kiang Liu. "Serum androgen concentrations in young men: A longitudinal analysis of associations with age, obesity, and race. The CARDIA male hormone study." Cancer Epidemiology, Biomarkers \& Prevention 11 (2002): 1041-47.

44. Zhihong Gong, Marian L. Neuhouser, Phyllis J. Goodman, Demetrius Albanes, Chen Chi, Ann W. Hsing, Scott M. Lippman, Elizabeth A. Platz, Michael N. Pollak, and Ian M. Thompson, et al. "Obesity, diabetes, and risk of prostate cancer: Results from the prostate cancer prevention trial.” Cancer Epidemiology, Biomarkers \& Prevention 15 (2006): 1977-83.

45. E. Spangler, C.M. Zeigler-Johnson, M. Coomes, S.B. Malkowicz, A. Wein, and T.R. Rebbeck. "Association of obesity with tumor characteristics and treatment failure of prostate cancer in African-American and European American men.” The Journal of Urology 178 (2007): 1939-45.

46. Megan Dann Fesinmeyer, Roman Gulati, Steve Zeliadt, Noel Weiss, Alan R. Kristal, and Ruth Etzioni. "Effect of population trends in body mass index on prostate cancer incidence and mortality in the United States.” Cancer Epidemiology, Biomarkers \& Prevention 18 (2009): 808-15.

47. Nina Føns Johnsen, Anne Tjønneland, Birthe L.R. Thomsen, Jane Christensen, Steffen Loft, Christine Friedenreich, Timothy J. Key, Naomi E. Allen, Petra H. Lahmann, and Lotte Mejlvig, et al. "Physical activity and risk of prostate cancer in the European Prospective Investigation into Cancer and Nutrition (EPIC) cohort.” International Journal of Cancer 125 (2009): 902-08.

48. Tom I.L. Nilsen, Pal R. Romundstad, and Lars J. Vatten. "Recreational physical activity and risk of prostate cancer: A prospective population-based study in Norway (the HUNT study)." International Journal of Cancer 119 (2006): 2943-47.

49. Karyn A. Esser, Clifford E. Harpole, and Gail S. Prins, and Alan M. Diamond. "Physical activity reduces prostate carcinogenesis in a transgenic model.” Prostate 69 (2009): 1372-77.

50. Rajagopalan Sridhar, Vernon Bond, Valerie Marie Cousins, Anwar Jackson, Richard Millis, and Renshu Zhang. “Aerobic capacity of African American males: Association with serum levels of IGF-1, IGF-1 binding protein and prostate cancer cell growth.” The Journal of the Federation of American Societies for Experimental Biology 23 (2009): 955.8 (Meeting Abstract Supplement).

51. Takashi Imamoto, Hiroyoshi Suzuki, Takanobu Utsumi, Takumi Endo, Makoto Takano, Masashi Yano, Koji Kawamura, Naoto Kamiya, Naoki Nihei, and Yukio Naya, et al. "Association between serum sex hormone levels and prostate cancer: Effect of prostate cancer on serum testosterone levels.” Future Oncology 5 (2009): 1005-13.

52. Hendrik Isbarn, Jehonathan H. Pinthus, Leonard S. Marks, Francesco Montorsi, Alvaro Morales, Abraham Morgentaler, and Claude Schulman. "Testosterone and prostate cancer: Revisiting old paradigms.” European Urology 56 (2009): 48-56.

53. Abraham Morgentaler. "Rapidly shifting concepts regarding androgens and prostate cancer." Scientific World Journal 9 (2009): 685-90.

54. Edward Giovannucci, Yan Liu, Elizabeth A. Platz, Meir J. Stampfer, and Walter C. Willett. "Risk factors for prostate cancer incidence and progression in the health professionals follow-up study.” International Journal of Cancer 121 (2007): 1571-78. 
55. Rajender Singh, Lalji Singh, and Kumarasamy Thangaraj. "Phenotypic heterogeneity of mutations in androgen receptor gene.” Asian Journal of Andrology 9 (2007): 147-79.

56. Ethan M. Lange, Aruna V. Sarma, Anna Ray, Yunfei Wang, Lindsey A. Ho, Sarah A. Anderson, Julie M. Cunningham, and Kathleen A. Cooney. "The androgen receptor CAG and GGN repeat polymorphisms and prostate cancer susceptibility in African-American men: Results from the Flint Men’s Health Study.” Journal of Human Genetics 53 (2008): 220-26.

57. Gary G. Schwartz. "Vitamin D and the epidemiology of prostate cancer." Seminars in Dialysis 18 (2005): 276-89.

58. Gary G. Schwartz, and Halcyon G. Skinner. "Vitamin D status and cancer: New insights." Currunt Opinion in Clinical Nutrition \&Metabolic Care 10 (2007): 6-11.

59. William B. Grant. "An estimate of premature cancer mortality in the U.S. due to inadequate doses of solar ultraviolet-B radiation.” Cancer 94 (2002): 1867-75.

60. Lois Y. Matsuoka, Jacobo Wortsman, John G. Haddad, Paul Kolm, and Bruce W. Hollis. "Racial pigmentation and the cutaneous synthesis of vitamin D.” JAMA Dermatology 127 (1991): 536-38.

61. Marilyn Tseng, Veda Giri, Deborah W. Bruner, and Edward Giovannucci. "Prevalence and correlates of vitamin D status in African American men.” BMC Public Health 9 (2009): 191.

62. Edward Giovannucci. "Vitamin D and cancer incidence in the Harvard cohorts." Annals of Epidemiology 19 (2009): 84-88.

63. Gary D. Steinberg, Bob S. Carter, Terri H. Beaty, Barton Childs, and Patrick C. Walsh. "Family history and the risk of prostate cancer.” Prostate 17 (1990): 337-47.

64. David C. Miller, Mark S. Litwin, Jonathan Bergman, Sevan Stepanian, Sarah E. Connor, Lorna Kwan, and William J. Aronson. "Prostate cancer severity among low income, uninsured men.” The Journal of Urology 181 (2009): 579-84.

65. Joel B. Braunstein, Noëlle S. Sherber, Steven P. Schulman, Eric L. Ding, and Neil R. Powe. "Race, medical researcher distrust, perceived harm, and willingness to participate in cardiovascular prevention trials.” Medicine 87 (2008): 1-9.

66. C. L. Bennett, M. R. Ferreira, T. C. Davis, J. Kaplan, M. Weinberger, T. Kuzel, M. A. Seday, and O. Sartor. "Relation between literacy, race, and stage of presentation among low-income patients with prostate cancer.” Journal of Clinical Oncology 16 (1998): 3101-04.

67. Gerald L. Andriole, E. David Crawford, Robert L. Grubb, Saundra S. Buys, David Chia, Timothy R. Church, Mona N. Fouad, Edward P. Gelmann, Paul A. Kvale, Douglas J. Reding, Joel L. Weissfeld, Lance A. Yokochi, Barbara O'Brien, Jonathan D. Clapp, Joshua M. Rathmell, Thomas L. Riley, Richard B. Hayes, Barnett S. Kramer, Grant Izmirlian, Anthony B. Miller, Paul F. Pinsky, Philip C. Prorok, John K. Gohagan, and Christine D. Berg, M.D. for the PLCO Project Team. "Mortality results from a randomized prostate-cancer screening trial.” The New England Journal of Medicine 360, no. 13 (2009): 1310-19.

68. U.S. Preventive Services Task Force. "Screening for prostate cancer: U.S. Preventive Services Task Force Recommendation Statement.” Annals of Internal Medicine 149, no. 3 (2008): 185-91.

69. AliciaK. Matthews, SarahA. Sellergren, Clara Manfredi, and Maryann Williams. "Factors influencing medical information seeking among African American cancer patients.” Journal of Health Communication 7 (2002): 205-19. 
70. Denethia B. Sellers, and Louie E. Ross. "African American men, prostate cancer screening and informed decision making.” Journal of the National Medical Association 95, no. 7 (2003): 618-25.

71. Deborah E. Blocker, LaHoma S. Romocki, Kamilah B. Thomas,Belinda L. Jones, Ethel Jean Jackson, LaVerne Reid, and Marci K. Campbell. "Knowledge, beliefs and barriers associated with prostate cancer prevention and screening behaviors among African-American men.” Journal of the National Medical Association 98, no. 8 (2006): 1286-95.

72. Michael A. Sanchez, Deborah J. Bowen, Alton. Hart, Jr, and Clarence Spigner. "Factors influencing prostate cancer screening decisions among African American men.” Ethnicity \& Disease 17, no. 2 (2007): 374-80.

73. C. Daniel Mullins, Eberechukwu Onukwugha, Kaloyan Bikov, Brian Seal, and Arif Hussain. "Health disparities in staging of SEER-Medicare prostate cancer patients in the United States." Urology 76 (2010): 566-72.

74. Nitya Abraham, Fei Wan, Chantal Montagnet, Yu-Ning Wong, and Katrina Armstrong. "Decrease in racial disparities in the staging evaluation for prostate cancer after publication of staging guidelines.” Journal of Urology 178 (2007): 82-87.

75. William V. Shappley, III, Stacey A. Kenfield, Julie L. Kasperzyk, Weiliang Qiu, Meir J. Stampfer, Martin G. Sanda, and June M. Chan. "Prospective study of determinants and outcomes of deferred treatment or watchful waiting among men with prostate cancer in a nationwide cohort.” Journal of Clinical Oncology 27 (2009): 4980-85.

76. Vickie L. Shavers, Martin L. Brown, Arnold L. Potosky, Carrie N. Klabunde, W.W. Davis, Judd W. Moul, and Angela Fahey. "Race/ethnicity and the receipt of watchful waiting for the initial management of prostate cancer.” Journal of General Internal Medicine 19 (2004): 146-55.

77. H. Ballentine Carter. “American Urological Association (AUA) Guideline on prostate cancer detection: Process and rationale.” BJU International 112, no. 5 (2013): 543-47.

78. Virginia A. Moyer. "Screening for prostate cancer: U.S. Preventive Services Task Force Recommendation Statement.” Annals of Internal Medicine 157, no. 2 (2012): 120-34.

79. Gary D. Steinberg, Gregory T. Bales and Charles B. Brendler. "An analysis of watchful waitingfor clinically localized prostate cancer.” The Journal of Urology 159, no. 5 (1998): 1431-36.

80. Julie L. Kasperzyk, William V. Shappley, III, Stacey A. Kenfield, Lorelei A. Mucci, Tobias Kurth, Jing Ma, Meir J. Stampfer, and Martin G. Sanda. "Watchful waiting and quality of life among prostate cancer survivors in the physicians' health study.” The Journal of Urology 186, no. 5 (2011): 1862-67.

81. Ping Tang, Leon Sun, Matthew A. Uhlman, Cary N. Robertson, Thomas J. Polascik, David M. Albala, Craig F. Donatucci, and Judd W. Moul. "Prostate-specific antigen-based risk-adapted discontinuation of prostate cancer screening in elderly African American and Caucasian American men.” Urology 76, no. 5 (2010): 1058-62.

82. Carrie N. Klabunde, Arnold L. Potosky, Linda C. Harlan, and Barnett S. Kramer. "Trends and black/ white differences in treatment for nonmetastatic prostate cancer.” Medical Care 36 (1998): 1337-48.

83. Kelvin A. Moses, Alan T. Paciorek, David F. Penson, Peter R. Carroll, and Viraj A. Master. "Impact of ethnicity on primary treatment choice and mortality in men with prostate cancer: Data from CaPSURE.” Journal of Clinical Oncology 28 (2010): 1069-74. 
84. Thomas D. Denberg Brenda L. Beaty, Fernando J. Kim, and John F. Steiner. "Marriage and ethnicity predict treatment in localized prostate carcinoma.” Cancer 103 (2005): 1819-25.

85. Nancy L. Keating, A. James O’Malley, Mary McNaughton-Collins, William K. Oh, and Matthew R. Smith. "Use of androgen deprivation therapy for metastatic prostate cancer in older men.” BJU International 101 (2008): 1077-83.

86. Kate McKeage, and Susan J. Keam. "Docetaxel in hormone-refractory metastatic prostate cancer.” Drugs 65 (2005): 2287-97.

87. Peter A. Humphrey. "Gleason grading and prognostic factors in carcinoma of the prostate." Modern Pathology 17 (2004): 292-306.

88. Ray M. Merrill, and Joseph L. Lyon. "Explaining the difference in prostate cancer mortality rates between white and black men in the United States.” Urology 55 (2000): 730-35.

89. Kendra Schwartz, Isaac J. Powell, Willie Underwood III, Julie George, Cecilia Yee, and Mousumi Banerjee. "Interplay of race, socioeconomic status, and treatment on survival of patients with prostate cancer.” Urology 74 (2009): 1296-302.

90. Tracy Onega, Eric J. Duell, Xun Shi, Eugene Demidenko, and David C. Goodman. "Race versus place of service in mortality among medicare beneficiaries with cancer.” Cancer 116 (2010): 2698-706.

91. Arthur A. Caire, Leon Sun, Thomas J. Polascik, David M. Albala, and Judd W. Moul. “Obese African-Americans with prostate cancer (T1c and a prostate-specific antigen, PSA, level of $<10 \mathrm{ng} / \mathrm{mL}$ ) have higher-risk pathological features and a greater risk of PSA recurrence than non-African-Americans.” BJU International 106 (2010): 1157-60.

92. Stephen J. Freedland, and Alan W. Partin. "Obesity and prostate cancer detection and progression.” Reviews in Urology 6 (2004): 214-16.

93. Susan Halabi, San-San Ou, Nicholas J. Vogelzang, and Eric J. Small. "Inverse correlation between body mass index and clinical outcomes in men with advanced castration-recurrent prostate cancer.” Cancer 110 (2007): 1478-84.

94. Carmen Rodriguez, Alpa V. Patel, Eugenia E. Calle, Eric J. Jacobs, Ann Chao, and Michael J. Thun. "Body mass index, height, and prostate cancer mortality in two large cohorts of adult men in the United States.” Cancer Epidemiology, Biomarkers \& Prevention 10 (2001): 345-53.

95. Christopher L. Amling. "The association between obesity and the progression of prostate and renal cell carcinoma.” Urologic Oncology 22 (2004): 478-84.

96. Stephen J. Freedland, William J. Aronson, Christopher J. Kane, Joseph C. Presti, Jr, Christopher L. Amling, David Elashoff, and Martha K. Terris. "Impact of obesity on biochemical control after radical prostatectomy for clinically localized prostate cancer: A report by the Shared Equal Access Regional Cancer Hospital database study group.” Journal of Clinical Oncology 22 (2004): 446-53.

97. Erin L Richman, Meir J. Stampfer, Alan Paciorek, Jeanette M. Broering, Peter R. Carroll, and June M. Chan. "Intakes of meat, fish, poultry, and eggs and risk of prostate cancer rogression.” The American Journal of Clinical Nutrition 91 (2010): 712-21.

98. Mansi Agarwal, Jill B. Hamilton, Charles E. Moore, and Jamie L. Crandell. "Predictors of depression among older African American cancer patients.” Cancer Nursing 33 (2010): 156-63. 
99. Chanita H. Halbert, James Coyne, Benita Weathers, Brandon Mahler, Ernestine Delmoor, David Vaughn, S. Bruce Malkowicz, David Lee, and Andrea Troxel. "Racial Differences in Quality of Life Following Prostate Cancer Diagnosis.” Urology 76 (2010): 559-64.

100. Barbara D. Powe, Jill Hamilton, Nichole Hancock, Natasha Johnson, Ramona Finnie, Jean Ko, Patrice Brooks, and Maurice Boggan, Jr. "Quality of life of African American cancer survivors. A review of the literature.” Cancer 109 (2007): 435-45.

101. Frank J. Penedo, Jason R. Dahn, Biing-Jiun Shen, Neil Schneiderman, and Michael H. Antoni. "Ethnicity and determinants of quality of life after prostate cancer treatment." Urology 67 (2006): 1022-27.

102. Deborah P. Lubeck, Howard Kim, Gary Grossfeld, Paul Ray, David F. Penson, Scott C. Flanders, and Peter R. Carroll. "Health related quality of life differences between black and white men with prostate cancer: Data from the cancer of the prostate strategic urologic research endeavor.” The Journal of Urology 166 (2001): 2281-85.

103. Jill B. Hamilton, Barbara D. Powe, Alton B. Pollard III, Karen J. Lee, and Alexandria M. Felton. "Spirituality among African American cancer survivors: Having a personal relationship with God.” Cancer Nursing 30 (2007): 309-16.

104. Marilyn J. Field, and Christine K. Cassel, eds. Approaching Death Improving Care at the End of Life. Washington: National Academy Press, 1997.

105. Kenneth A. Wallston, Candice Burger, Roberta Ann Smith, and Robert J. Baugher. "Comparing the quality of death for hospice and non-hospice cancer patients.” Medical Care 26 (1988): 177-82.

106. Jennifer S. Haas, Craig C. Earle, John E. Orav, Phyllis Brawarsky, Bridget A. Neville, Dolores Acevedo-Garcia, and David R. Williams. "Lower use of hospice by cancer patients who live in minority versus white areas.” Journal of General Internal Medicine 22 (2007): 396-99.

107. Joshua R. Gonzalez, Nathan S. Consedine, James M. McKiernan, and Benjamin A. Spencer. "Barriers to the initiation and maintenance of prostate specific antigen screening in black American and Afro-Caribbean men.” Journal of Urology 180 (2008): 2403-08.

108. Nathan S. Consedine, Brenda A. Adjei, Paul M. Ramirez, and James M. McKiernan. “An object lesson: Source determines the relations that trait anxiety, prostate cancer worry, and screening fear hold with prostate screening frequency.” Cancer Epidemiology, Biomarkers and Prevention 17 (2008): 1631-39.

109. Daniel J. Lee, Nathan S. Consedine, and Benjamin A. Spencer. "Barriers and facilitators to digital rectal examination screening among African-American and African-Caribbean men.” Urology 77 (2011): 891-98.

110. Nathan S. Consedine, Amy H. Morgenstern, Elizabeth Kudadjie-Gyamfi,Carol Magai, and Alfred I. Neugut. "Prostate cancer screening behavior in men from seven ethnic groups: The fear factor.” Cancer Epidemiology, Biomarkers \& Prevention 15 (2006): 228-37.

111. Adrienne A. Phillips, Judith S. Jacobson, Carol Magai, Nathan Consedine, Nathalie C. Horowicz-Mehler, and Alfred I Neugut. "Cancer incidence and mortality in the Caribbean." Cancer Investigation 25 (2007): 476-83.

112. Chris F. Heyns, Angus T. Lecuona, and Grant S. Trollip. "Prostate cancer: Prevalence and treatment in African men.” The Journal of Men's Health \& Gender 2, no. 4 (2005): 400-05. 
113. Lisa W. Chu, Jamie Ritchey, and Susan S. Devesa, et al. "Prostate cancer incidence rates in Africa.” Prostate Cancer 2011, Article ID 947870. http://dx.doi.org/10.1155/2011/947870.

114. Yoav Ben-Shlomo, Simon Evans, Fowzia Ibrahim,Biral Patel, Ken Anson, Frank Chinegwundoh, Cathy Corbishley, Danny Dorling, Bethan Thomas, and David Gillatt. "The Risk of prostate cancer amongst black men in the United Kingdom: The PROCESS Cohort Study.” European Urology 53, no. 1 (2008): 99-105.

(C) 2013 by the authors; licensee MDPI, Basel, Switzerland. This article is an open access article distributed under the terms and conditions of the Creative Commons Attribution license (http://creativecommons.org/licenses/by/3.0/). 